\title{
A Surprisingly Stable S-Nitrosothiol Complex
}

\author{
Supporting information
}

Laura L. Perissinotti ${ }^{\dagger}$, Darío A. Estrin ${ }^{\dagger}$, Gregory Leitus ${ }^{\ddagger}$ and Fabio Doctorovich ${ }^{\star \dagger}$

${ }^{\dagger}$ Departamento de Química Inorgánica, Analítica y Química Física / INQUIMAE, Facultad de Ciencias Exactas y Naturales, Universidad de Buenos Aires. Ciudad Universitaria, Pabellón II, (C1428EHA) Buenos Aires, Argentina.

${ }^{\ddagger}$ Department of Chemical Services, The Weizmann Institute of Science, Rehovot 76100, Israel. 


\section{Materials and methods}

Materials. Benzyl mercaptan $\left(\mathrm{PhCH}_{2} \mathrm{SH}\right)$ was purchased from Fluka, $\mathrm{K}\left[\mathrm{IrCl}_{6}\right], \mathrm{K}\left[\mathrm{IrCl}_{5}(\mathrm{NO})\right]$ and $\mathrm{Na}^{15} \mathrm{NO}_{2}$ were purchased from Aldrich. Acetonitrile was purchased from Aldrich; it was dried and distilled. DMSO and $\mathrm{D}_{2} \mathrm{O}$ were purchased from Aldrich and used as received. The water used in all reactions was MilliQ Water obtained from deionized water.

Synthesis of Trans-K[ $\left.\mathrm{IrCl}_{4}\left(\mathrm{CH}_{3} \mathrm{CN}\right) \mathrm{N}(\mathrm{O}) \mathrm{SCH}_{2} \mathrm{Ph}\right](3): 27 \mu \mathrm{L}\left(2.28 \times 10^{-2} \mathrm{mmol}\right)$ of $\mathrm{PhCH}_{2} \mathrm{SH}$ (dissolved in $0.25 \mathrm{~mL}$ of acetonitrile) were added under $\mathrm{Ar}$ atmosphere to $10 \mathrm{mg}\left(2.28 \times 10^{-2} \mathrm{mmol}\right)$ of $\mathrm{K}\left[\mathrm{IrCl}_{5}(\mathrm{NO})\right]$ in $0.25 \mathrm{~mL}$ of acetonitrile. The product which precipitated was separated from the solution by centrifugation, the solid was dried under vacuum. Reaction yield: $80 \%$. Red crystals were obtained by slow evaporation of the supernatant.

Trans- $\left.\mathrm{K}\left[\mathrm{IrCl}_{4}\left(\mathrm{CH}_{3} \mathrm{CN}\right)^{15} \mathrm{~N}(\mathrm{O}) \mathrm{SCH}_{2} \mathrm{Ph}\right]\right]$ was prepared in an analogous manner, using $\mathrm{K}\left[\operatorname{IrCl}_{5}\left({ }^{15} \mathrm{NO}\right)\right]$ prepared from $\mathrm{Na}^{15} \mathrm{NO}_{2}$ and $\mathrm{K}\left[\operatorname{IrCl}_{6}\right]$.

NMR Spectroscopy. NMR spectra were acquired on a Bruker AM 500 spectrometer. All measurements were carried out in $\mathrm{D}_{2} \mathrm{O}$.

UV/Visible Spectroscopy. Absorption spectra were acquired on a Hewlett-Packard HP8453 diode array spectrometer.

FTIR Spectroscopy. FTIR spectra were acquired with Nicolet Avatar FTIR spectrophotometer. Solid spectra were obtained as $\mathrm{KBr}$ pellets .

Mass Spectrometry. 3 was analyzed by negative mode (NM) Q-TOF-ESI-MS, we also studied 3 by ESI-MS-MS of the principal ions. The analyzed products with the general formula: $\left[\operatorname{IrCl}_{5} \mathrm{~N}(\mathrm{O}) \mathrm{SR}\right]^{2-}$ (with $\mathrm{R}=$ benzyl; counterions $\mathrm{K}+$ and $\mathrm{H}+$ ) showed by MS NM the ions [M-K-H-Cl] ${ }^{-}$(m/z:485.86), [M $\mathrm{H}]^{-}\left(\mathrm{m} / \mathrm{z}: 561.7\right.$ ), and [M-K-Cl-H-NO] ${ }^{-}(\mathrm{m} / \mathrm{z}: 455.87)$.

Theoretical Calculations. All the calculations performed in this work were carried out using the Gaussian $98^{1}$ package. We have fully optimized the geometries of all species at the BPW $91^{2}$ level with a 6-31G** basis set for the first row atoms, combined with quasirelativistic electron core potentials $\left(\mathrm{LANL}_{2} \mathrm{DZ}^{3}\right.$ ) and the corresponding optimized sets of basis functions for the iridium center. Each stationary point in the gas phase was characterized by performing a normal modes analysis.

\section{X-Ray, Crystal data:}

Table SI 1. Crystal data and structure refinement for $3 . \mathrm{CH}_{3} \mathrm{CN}$.

$\begin{array}{lc}\text { Identification code } & \mathrm{m} 592 \mathrm{a} \\ \text { Empirical formula } & \mathrm{C} 11 \mathrm{H} 13 \mathrm{Cl} \text { Ir K N3 O S } \\ \text { Formula weight } & 608.40 \\ \text { Temperature } & 120(2) \mathrm{K} \\ \text { Wavelength } & 0.71073 \mathrm{~A} \\ \text { Crystal system, space group } & \text { Monoclinic, P2(1)/n } \\ \text { Unit cell dimensions } & \mathrm{a}=17.548(4) \mathrm{A} \text { alpha }=90 \mathrm{deg} . \\ & \mathrm{b}=6.8190(14) \mathrm{A} \text { beta }=115.40(3) \mathrm{deg} . \\ & \mathrm{c}=18.261(4) \mathrm{A} \text { gamma }=90 \mathrm{deg} . \\ \text { Volume } & 1973.8(7) \mathrm{A}^{3}\end{array}$

$\begin{array}{lc}\text { Z, Calculated density } & 4,2.047 \mathrm{Mg} / \mathrm{m}^{3} \\ \text { Absorption coefficient } & 7.624 \mathrm{~mm}^{-1} \\ \mathrm{~F}(000) & 1152 \\ \text { Crystal size } & 0.30 \times 0.30 \times 0.02 \mathrm{~mm} \\ \text { Theta range for data collection } 2.47 \text { to } 20.81 \mathrm{deg} . \\ \begin{array}{ll}\text { Limiting indices } & -17 \leq \mathrm{h} \leq 15,0 \leq \mathrm{k} \leq 6,0 \leq \mathrm{l} \leq 18\end{array}\end{array}$




$\begin{array}{lc}\text { Reflections collected / unique } & 2061 / 2061 \text { [R(int })=0.0000] \\ \text { Completeness to theta }=20.81 & 99.8 \% \\ \text { Max. and min. transmission } & 0.8625 \text { and } 0.2082 \\ \text { Refinement method } & \text { Full-matrix least-squares on } \mathrm{F}^{2} \\ \text { Data / restraints / parameters } & 2061 / 0 / 196 \\ \text { Goodness-of-fit on F^2 } & 1.167 \\ \text { Final R indices [I }>2 \text { sigma(I)] } & \mathrm{R} 1=0.0370, \mathrm{wR} 2=0.0670 \\ \text { R indices (all data) } \quad \mathrm{R} 1=0.0447, \mathrm{wR} 2=0.0691 \\ \begin{array}{ll}\text { Largest diff. peak and hole } & 0.647 \text { and }-0.772 \mathrm{e}^{-3}\end{array}\end{array}$

Table SI 2. Atomic coordinates $\left(x 10^{4}\right)$ and equivalent isotropic displacement parameters $\left(\mathrm{A}^{2} \times 10^{3}\right)$ for m592a. $\mathrm{U}(\mathrm{eq})$ is defined as one third of the trace of the orthogonalized Uij tensor.

\begin{tabular}{|c|c|c|c|c|}
\hline & $\mathrm{x}$ & $\mathrm{z}$ & $\mathrm{U}(\mathrm{eq})$ & \\
\hline $\operatorname{Ir}(1)$ & $1758(1)$ & $8026(1)$ & $91(1)$ & $14(1)$ \\
\hline $\mathrm{Cl}(1)$ & $1944(2)$ & $10853(4)$ & $-546(1)$ & $18(1)$ \\
\hline $\mathrm{Cl}(2)$ & $3214(2)$ & $7824(4)$ & $875(1)$ & $19(1)$ \\
\hline $\mathrm{Cl}(3)$ & $278(1)$ & $8268(4)$ & $-685(1)$ & $17(1)$ \\
\hline $\mathrm{Cl}(4)$ & $1540(2)$ & $5246(4)$ & $749(1)$ & $17(1)$ \\
\hline $\mathrm{N}(1)$ & $1814(5)$ & $6336(11)$ & $-752(4)$ & $9(2)$ \\
\hline $\mathrm{O}(1)$ & $1254(4)$ & $5228(9)$ & $-1175(4)$ & $17(2)$ \\
\hline$S(1)$ & $2717(2)$ & $6388(4)$ & $-918(2)$ & $20(1)$ \\
\hline$C(1)$ & $2431(6)$ & $4575(16)$ & $-1720(6)$ & $23(3)$ \\
\hline $\mathrm{C}(2)$ & $3219(6)$ & $3824(15)$ & $-1743(6)$ & $16(3)$ \\
\hline$C(3)$ & $3711(7)$ & $2417(17)$ & $-1200(6)$ & $33(3)$ \\
\hline $\mathrm{C}(4)$ & $4414(8)$ & $1660(20)$ & $-1245(7)$ & $47(4)$ \\
\hline $\mathrm{C}(5)$ & $4634(7)$ & 2292(18) & $-1854(7)$ & $35(3)$ \\
\hline$C(6)$ & $4163(7)$ & $3645(19)$ & $-2399(7)$ & $35(3)$ \\
\hline$C(7)$ & $3459(7)$ & $4449(16)$ & $-2349(6)$ & $24(3)$ \\
\hline $\mathrm{N}(2)$ & 1707(5) & $9741(12)$ & $982(5)$ & $12(2)$ \\
\hline $\mathrm{C}(8)$ & $1734(6)$ & $10617(15)$ & $1524(7)$ & $17(3)$ \\
\hline $\mathrm{C}(9)$ & 1794(6) & $11745(15)$ & $2214(5)$ & $20(3)$ \\
\hline $\mathrm{N}(3)$ & $811(7)$ & $7398(17)$ & $2420(6)$ & $52(3)$ \\
\hline$C(10)$ & $935(7)$ & $7588(17)$ & $3077(8)$ & $37(3)$ \\
\hline $\mathrm{C}(11)$ & $1066(8)$ & $7854(17)$ & $3916(6)$ & 43(3) \\
\hline $\mathrm{K}(1)$ & $-178(1)$ & $7051(4)$ & $754(1)$ & 21(1) \\
\hline
\end{tabular}

Table SI 3. Cartesian coordinates from BPW91/ LANL2DZ optimizations:

\section{Product (3)}

\begin{tabular}{|c|c|c|c|c|}
\hline \multirow{2}{*}{$\begin{array}{l}\text { Center } \\
\text { Number }\end{array}$} & \multirow{2}{*}{$\begin{array}{l}\text { Atomic } \\
\text { Number }\end{array}$} & \multicolumn{3}{|c|}{ Coordinates (Angstroms) } \\
\hline & & X & $\mathrm{Y}$ & $\mathrm{Z}$ \\
\hline 1 & 77 & 3.192707 & 5.385767 & 0.175943 \\
\hline 2 & 17 & 3.519102 & 7.482691 & -0.989626 \\
\hline 3 & 17 & 5.369299 & 5.634321 & 1.206859 \\
\hline 4 & 17 & 0.962689 & 5.220977 & -0.737619 \\
\hline 5 & 17 & 2.825907 & 3.367149 & 1.452425 \\
\hline 6 & 7 & 3.898374 & 4.334477 & -1.307084 \\
\hline
\end{tabular}




$\begin{array}{ccccc}7 & 8 & 3.427089 & 3.301351 & -1.742021 \\ 8 & 16 & 5.448432 & 4.997234 & -2.114018 \\ 9 & 6 & 5.727340 & 3.652800 & -3.314001 \\ 10 & 1 & 4.821750 & 3.009113 & -3.205383 \\ 11 & 1 & 5.708885 & 4.090043 & -4.331313 \\ 12 & 6 & 6.992760 & 2.857094 & -3.086697 \\ 13 & 6 & 7.229074 & 2.193187 & -1.864085 \\ 14 & 1 & 6.499107 & 2.289251 & -1.045717 \\ 15 & 6 & 8.390458 & 1.432706 & -1.679851 \\ 16 & 1 & 8.556100 & 0.921554 & -0.718832 \\ 17 & 6 & 9.343334 & 1.325845 & -2.707397 \\ 18 & 1 & 10.258058 & 0.731272 & -2.556594 \\ 19 & 6 & 9.123606 & 1.989563 & -3.923014 \\ 20 & 1 & 9.866097 & 1.920845 & -4.733763 \\ 21 & 6 & 7.957283 & 2.749556 & -4.107329 \\ 22 & 1 & 7.789381 & 3.271386 & -5.063709 \\ 23 & 7 & 2.420716 & 6.418846 & 1.705510 \\ 24 & 6 & 1.958335 & 7.003601 & 2.597105 \\ 25 & 6 & 1.376629 & 7.734002 & 3.709458 \\ 26 & 1 & 0.865692 & 8.644571 & 3.337882 \\ 27 & 1 & 2.168837 & 8.034755 & 4.423726 \\ 28 & 1 & 0.637958 & 7.099043 & 4.238146 \\ & & & & \end{array}$

\section{$\mathrm{PhCH}_{2}$-SNO}

\begin{tabular}{|c|c|c|c|c|}
\hline \multirow{2}{*}{$\begin{array}{l}\text { Center } \\
\text { Number }\end{array}$} & \multirow{2}{*}{$\begin{array}{l}\text { Atomic } \\
\text { Number }\end{array}$} & \multicolumn{3}{|c|}{ Coordinates (Angstroms) } \\
\hline & & $\mathrm{X}$ & $\mathrm{Y}$ & $\mathrm{Z}$ \\
\hline 1 & 6 & 0.035343 & -0.042119 & -0.185324 \\
\hline 2 & 6 & -0.154430 & -0.007783 & 1.209990 \\
\hline 3 & 6 & 0.978504 & 0.033189 & 2.047870 \\
\hline 4 & 6 & 2.269172 & 0.039338 & 1.502568 \\
\hline 5 & 6 & 2.447795 & 0.006332 & 0.110337 \\
\hline 6 & 6 & 1.327026 & -0.035789 & -0.732279 \\
\hline 7 & 6 & -1.547332 & -0.010803 & 1.792530 \\
\hline 8 & 16 & -2.130302 & 1.646269 & 2.295850 \\
\hline 9 & 7 & -2.485395 & 1.386276 & 4.144807 \\
\hline 10 & 8 & -2.292774 & 0.292712 & 4.586704 \\
\hline 11 & 1 & -1.603685 & -0.619279 & 2.726591 \\
\hline 12 & 1 & -2.287406 & -0.418030 & 1.076826 \\
\hline 13 & 1 & 0.846429 & 0.057835 & 3.140962 \\
\hline 14 & 1 & 3.143809 & 0.066953 & 2.170133 \\
\hline 15 & 1 & 3.461909 & 0.010799 & -0.317359 \\
\hline 16 & 1 & 1.458106 & -0.065363 & -1.824683 \\
\hline 17 & 1 & -0.841243 & -0.077388 & -0.851736 \\
\hline
\end{tabular}

Table SI 4. NBO charge calculation, BPW91/ LANL2DZ

\begin{tabular}{ccccc}
\hline & $\mathrm{Ir}$ & $\mathrm{N}$ & $\mathrm{O}$ & $\mathrm{S}$ \\
\hline $\mathbf{3}$ & +0.684 & +0.033 & -0.254 & +0.416 \\
$\mathrm{PhCH}_{2}-\mathrm{SNO}$ & & +0.060 & -0.242 & +0.272 \\
\hline
\end{tabular}


Figure SI 1. UV-visible spectrum of $3\left(2.58 \times 10^{-4} \mathrm{M}\right)$ in acetonitrile.

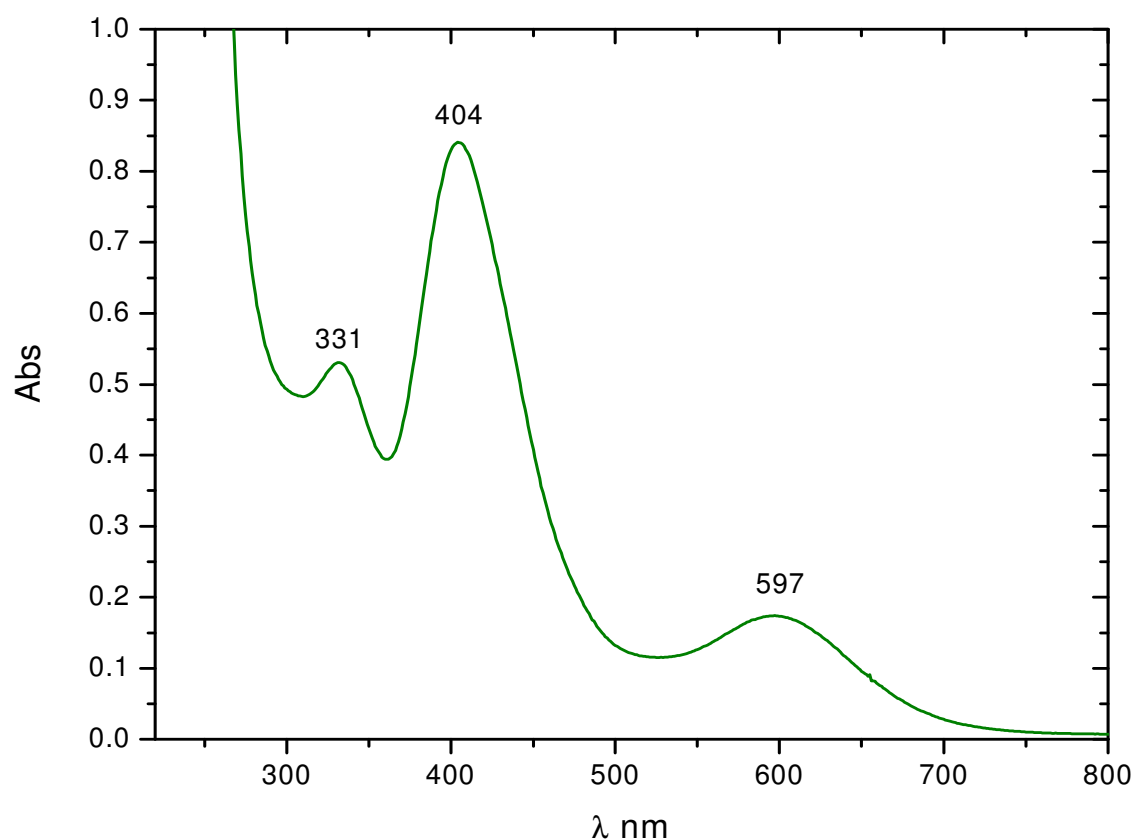


Figure SI 2. ${ }^{1} \mathrm{H}$ NMR spectra for $\mathbf{1}$.
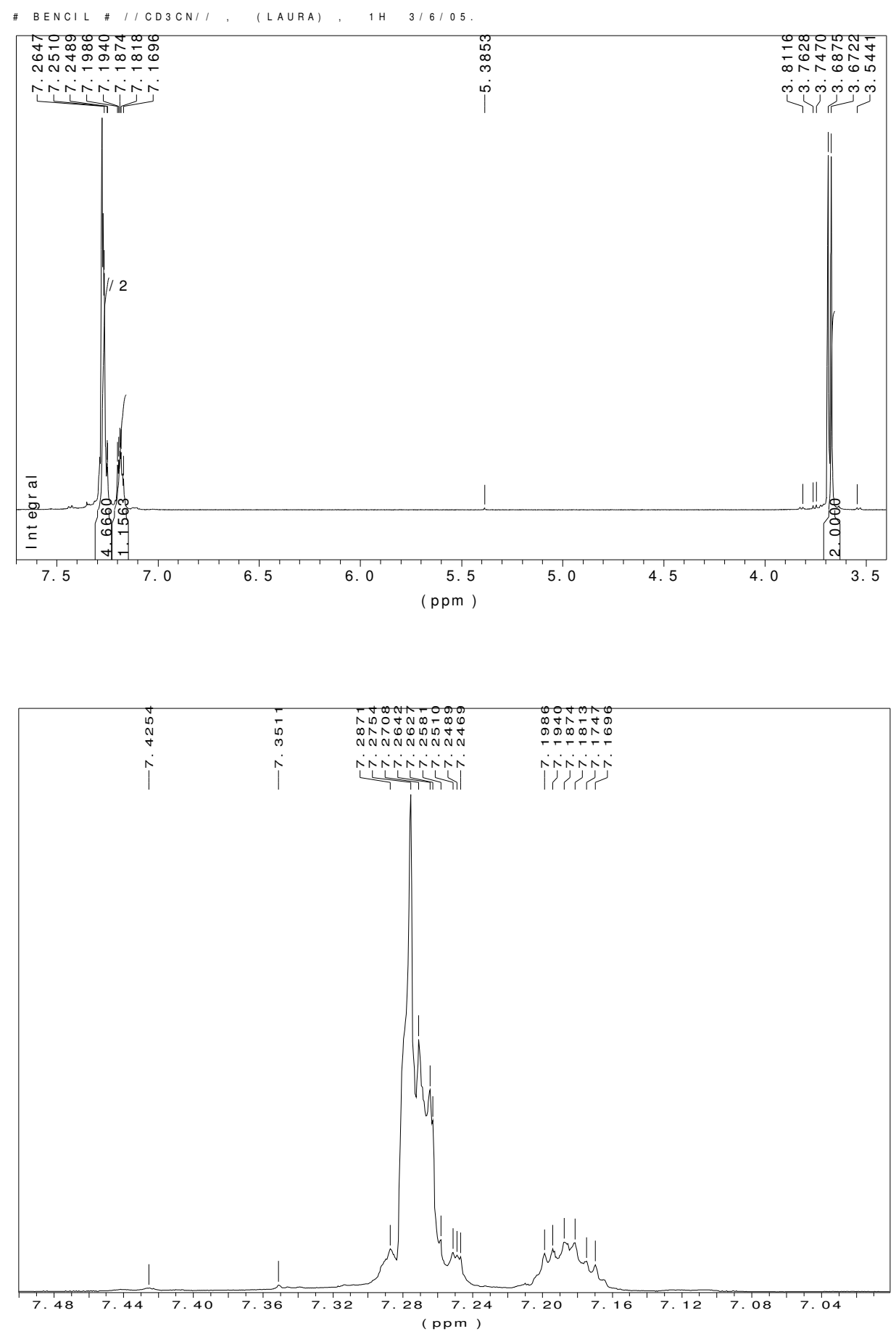

\section{${ }^{1}$ H NMR assignment:}

$\delta 3.6$ (doublet,2H, $\mathbf{C H}_{2}$ ); $\delta$ 7.16-7.18 (multiplet, $1 \mathrm{H}, \mathrm{Ar}-\mathbf{H}$ ) $; \delta$ 7.19-7.26 (multiplet, 4H, Ar-H) 
Figure SI 3. ${ }^{1} \mathrm{H}$ NMR spectra for 3 .
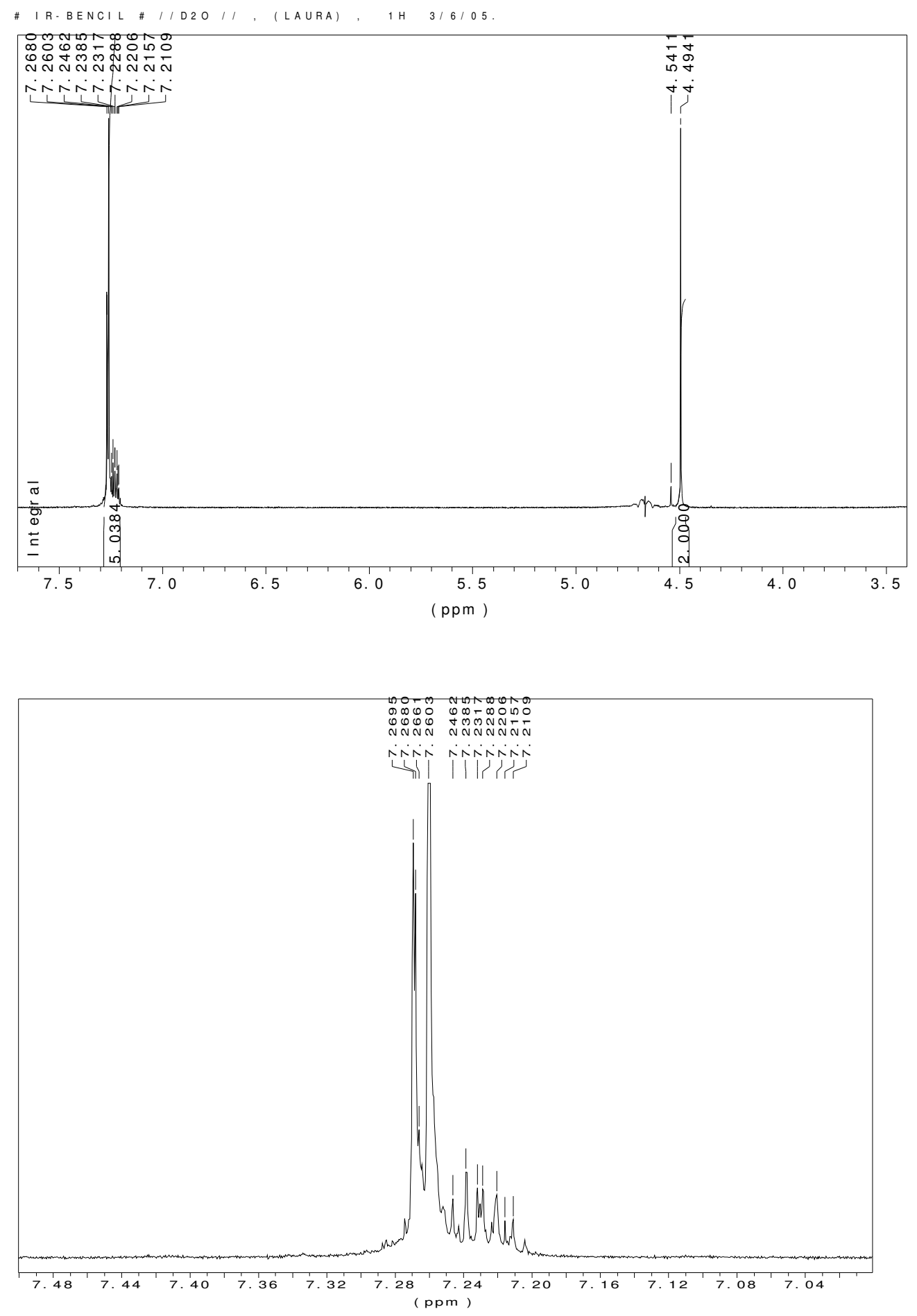

${ }^{1} \mathrm{H}$ NMR assignment:

$\delta 4.5$ (doublet,2H, $\mathbf{C H}_{2}$ ); $\delta$ 7.16-7.18 (multiplet, $1 \mathrm{H}, \mathrm{Ar}-\mathbf{H}$ ) $; \delta$ 7.19-7.26 (multiplet, $4 \mathrm{H}$, Ar-H). 
Figure SI 4. Complete FTIR spectra (Solid State) for the unlabeled (blue) and ${ }^{15} \mathrm{~N}$ isotopically labelled (red) complex and difference spectra (magenta).

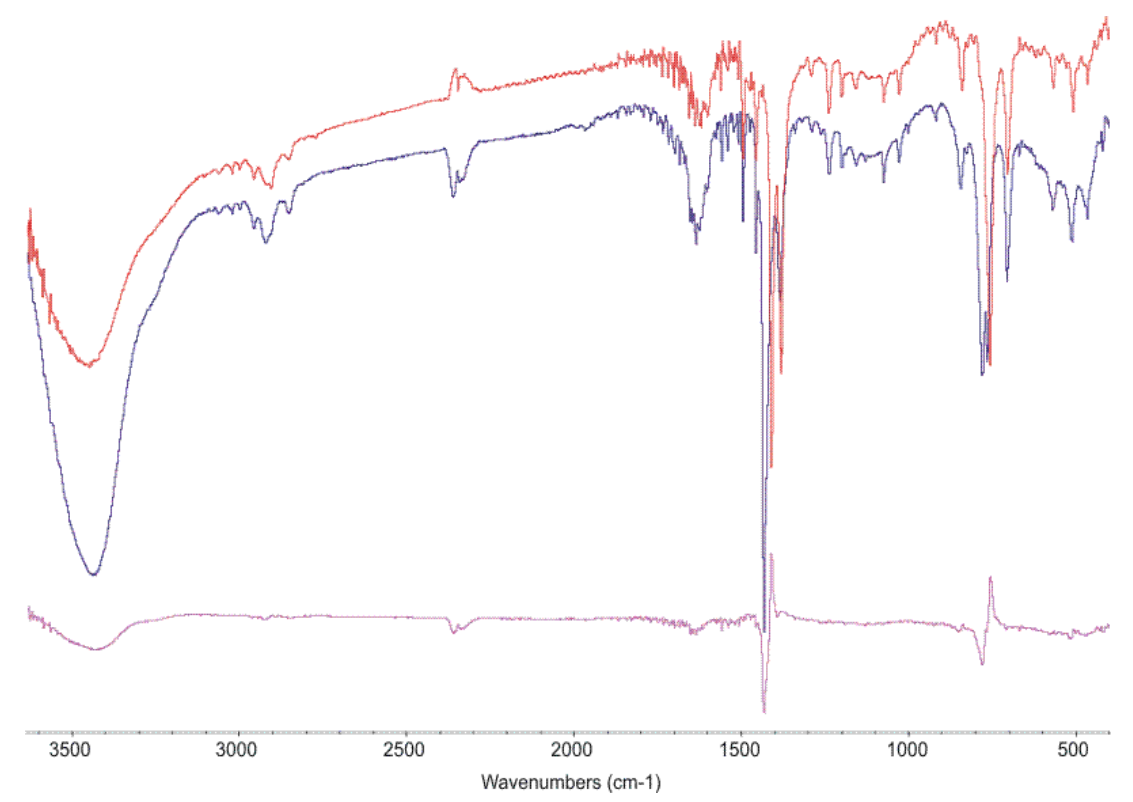

Figure SI 5. FTIR spectra for 3 in $\mathrm{D}_{2} \mathrm{O}$.

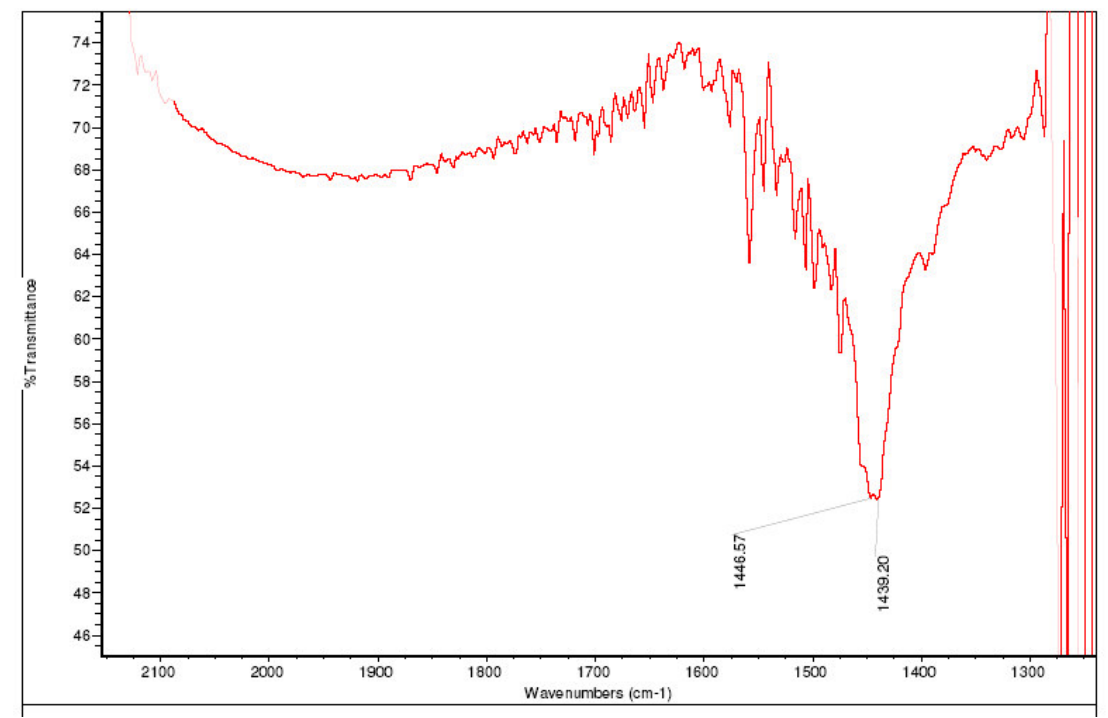

\section{${ }^{1}$ Complete Gaussian98 citation:}

Frisch, M. J.; Trucks, G. W.; Schlegel, H. B.; Scuseria, G. E.; Robb, M. A.; Cheeseman, J. R.; Zakrzewski, V. G.; Montgomery, J. A. Jr.; Stratmann, R.; Burant, J.; Dapprich, S.; Millam, J. M.; Daniels, A. D.; Kudin, K. N.; Strain, M. C.; Farkas, O.; Tomasi, J.; Barone, V. ; Cossi, M.; Cammi, R.; Mennucci, B.; Pomelli, C.; Adamo, C.; Clifford, S.; Ochterski,J.; Petersson, G. A.; Ayala, P. Y.; Cui, Q.; Morokuma, K.; Malick, D. K.; Rabuck, A. D.; Raghavachari, K.; Foresman, J. B.; Cioslowski, J.; Ortiz, J. V.; Baboul, A. G.; Stefanov, B. B.; Liu, G.; Liashenko, A.; Piskorz, P.; Komaromi, I.; Gomperts, R.; Martin, R. L.; Fox, D. J.; Keith, T.; Al-Laham, M. A.; Peng, C. Y.; Nanayakkara, A.; Gonzalez, C.; Challacombe, M.; Gill, P. M. W.; Johnson, B.; Chen, W.; Wong, M. W.; Andres, J. L.; Gonzalez, C.; Head-Gordon, M.; Replogle, E. S.; and Pople, J. A. , Gaussian 98, Rev. A7, Gaussian, Inc., Pittsburgh PA, 1998.

2 (a) Becke, A. D. Phys. Rev. A, 1988, 38, 3098-3100. (b) Perdew, J. P.; Wang, Y. Phys. Rev. B 1992, 45, 1324413249.

3 Hay, P., J.: Wadt, W., R. J. Chem. Phys. 1985, 82, 299-310. 